\title{
Barrett's esophagus
}

B ARRETT'S ESOPHAGUS IS DEFINED AS A COLUMNAR-LINED esophagus that develops when previously damaged squamous mucosa in the distal esophagus is replaced by metaplastic columnar mucosa. Almost invariably gastroesophageal reflux is the factor that injures the squamous mucosa. However, rare causes of Barrett's esophagus include radiation damage and alkaline reflux damage after total gastrectomy. Esophageal columnar metaplastic mucosa consists of one type or a combination of up to three types of columnar epithelia: fundal, junctional and specialized columnar (a variant of incomplete intestinal metaplasia). It is this so-called 'intestinal-type' that appears to be associated with the development of dysplasia and adenocarcinoma. It is likely that the intestinal-type mucosa is derived from the esophageal submucosal glands, presumably from stem cells capable of such change.

What is the effect of the components of gastric juice on Barrett's mucosa? There have been several studies that have documented abnormal components of gastric juice, such as bile acids from duodenogastric reflux, in patients with Barrett's esophagus. However, the evidence at this time is equivocal as to whether alkaline reflux or bile acids reflux play a role in the development and perpetuation of this mucosal change. There is one oft' quoted experiment where, after gastrectomy, reflux of duodenal content into the mucosa of rats is followed by the development of columnar-lined esophagus, dysplasia and eventually carcinoma. The esophageal change was premalignant, and when a carcinogen was added to the rat model, $50 \%$ of the animals developed carcinoma. In humans, esophagogastrostomy results in significant reflux and at least half the patients eventually go on to develop columnar-lined epithelium from the continued gastroesophageal reflux. This operation is rarely performed now.

Does Helicobacter pylori infection occur in Barrett's esophagus? H pylori is the causal agent of type B gastritis, and an association of $H$ pylori infection in gastric B cancerous lesions has also been established. Thus, $H$ pylori infection may be central in the sequence of events leading to gastric cancer. Review of the literature shows that the relationship between $\mathrm{H}$ pylori and esophageal pathology is inconstant and ranges from 0 to $52 \%$, with most studies showing less than $20 \%$ prevalence in these patients. Any causal relationship between the presence of the organism and the subsequent dysplastic and carcinomatous change remains speculative.

The endoscopic diagnosis is usually straightforward but can be very difficult. The junction of the squamous and columnar mucosa - the $\mathrm{Z}$ line - should coincide with the esophagogastric junction at the lower border of the lower esophageal sphincter. This esophagogastric junction is defined endoscopically as the proximal extent of the gastric fold. However, there are normal variations, such as that the distal 2 to $3 \mathrm{~cm}$ of the esophagus may be lined in whole or in part by columnar epithelium. Consequently, columnar metaplasia may be present only when the squamocolumnar mucosal junction is situated more than 2 to $3 \mathrm{~cm}$ proximal to the proximal extent of the gastric folds. There may be short tongues or flame-shaped segments or patches, which if they contain intestinal-type mucosa on biopsy, confirm the diagnosis of Barrett's esophagus. The colour change associated with columnar metaplasia is easily recognized as a classic

Gastroenterology in the '90s is a series of short articles addressing topical, controversial issues in the treatment of gastrointestinal disorders. The series is made possible through an educational grant from Astra Pharma Inc salmon pink, contrasting with the paler esophageal squamous epithelium. Multiple biopsies around the circumference of the Barrett's esophagus are necessary to define its presence, extent of change and any dysphagia. The endoscopic and histological appearance of Barrett's mucosa in children does not differ from that in adults.

Other diagnostic modalities have been assessed in the diagnosis of Barrett's esophagus. Occasionally barium swallow will show a reticular mucosal pattern, which when present is a relatively specific radiologic criterion for Barrett's esophagus, particularly if located adjacent to the distal aspect of the highest stricture. Similarly, a radionuclide scan of Barrett's esophagus using ${ }^{99 \mathrm{M}} \mathrm{Tc}$ pertechnate uptake may occasionally be diagnostic. These findings do not justify these investigations in the diagnosis of Barrett's esophagus.

Symptomatically, patients with Barrett's esophagus are indistinguishable from those with gastroesophageal reflux symptoms who do not have Barrett's change. Consequently, the diagnosis can only be made by endoscopic biopsy.

The significance of Barrett's esophagus is the linked propensity to develop esophageal cancer, estimated to be between 30 and 125 times greater in those with the disease than the incidence in the general population. Recent North American population studies have documented an increasing incidence of adenocarcinoma of the esophagus, which when limited to the esophagogastric junction, is approximately two per 100,000 per year.

Should we be performing a screening program? In Olmstead County, Minnesota, an analysis was not cost-effective - estimated to cost over $\$ 1$ million to prevent one death from Barrett's esophagusrelated adenocarcinoma. Consequently, routine screening is not recommended.

The complications of the columnar lining of Barrett's esophagus include the development of stricture, ulceration, bleeding and adenocarcinoma. Very rarely, spontaneous esophageal perforation of a Barrett's ulcer has been described. Studies have refuted the argument that hypersecretion of acid in the columnar-lined esophagus takes place and that it is a significant factor in the development of these complications.

The treatment of Barrett's esophagus is controversial. Surgical repair of gastroesophageal reflux leads to reversal of Barrett's change, and the corollary of incomplete operation with recurrent reflux reveals that Barrett's change may progress. The use of proton pump inhibitors such as omeprazole has been shown to ameliorate the changes of Barrett's esophagus. However, appropriate, long term, controlled studies have yet to be reported. The use of controlled laser therapy in esophageal dysplastic epithelium is receiving increasing attention.

\section{SUGGESTED READING}

Barrett NR. Surgery 1957;41:881-94.

Achkar E, Carey W. Am J Gastroenterol 1988;83:291-4.

Cameron AJ, Lomboy CT. Gastroenterology 1992;103:1241-5.

Gillen T, et al. Br J Surg 1988;75:540-3.

Giuli R, et al, eds. The Esophageal Mucosa. Amsterdam: Elsevier, 1994

Robertson CS, et al. Br J Surg 1988;75:760-3.

Spechler SJ. Gastroenterology 1993;104:1855-8.

Tytgat GNJ, et al. Endoscopy 1989;21:177-85

CN Williams MD FRCPC Halifax, Nova Scotia 


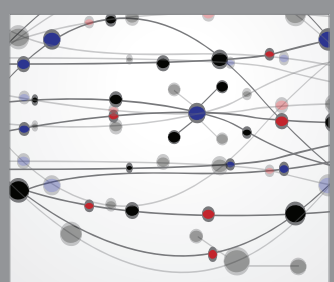

The Scientific World Journal
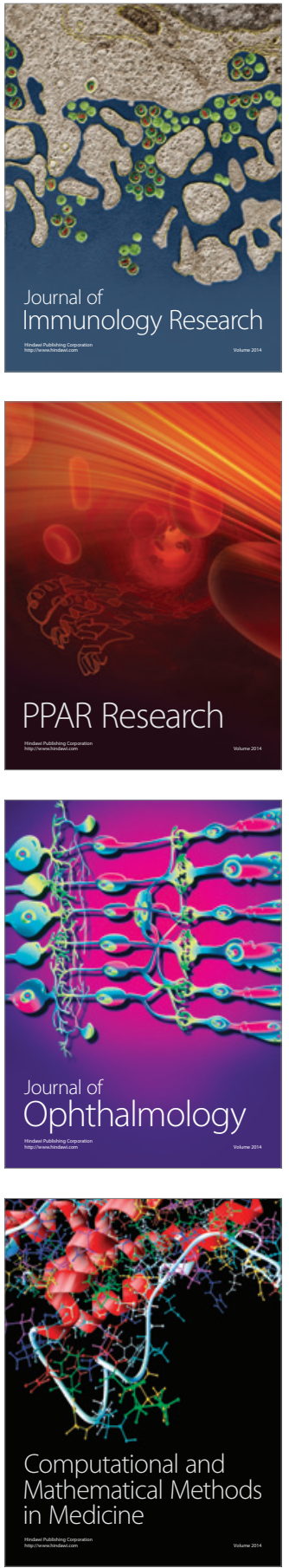

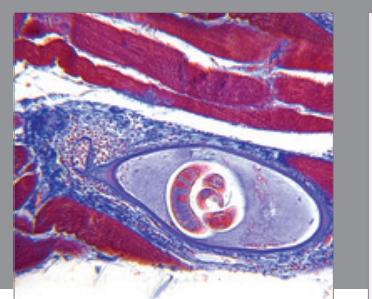

Gastroenterology Research and Practice

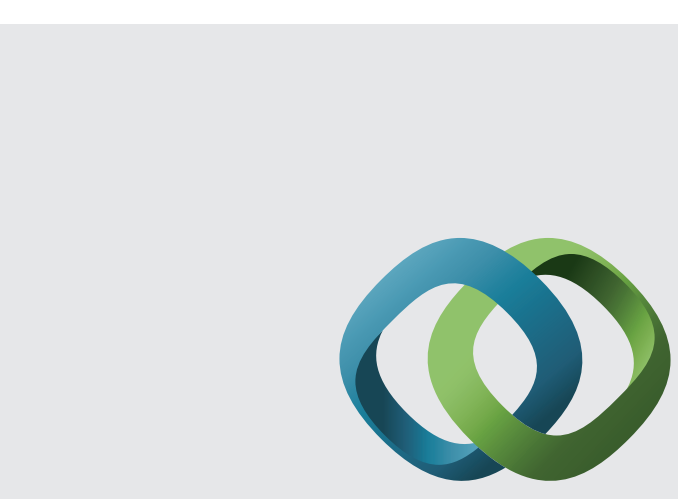

\section{Hindawi}

Submit your manuscripts at

http://www.hindawi.com
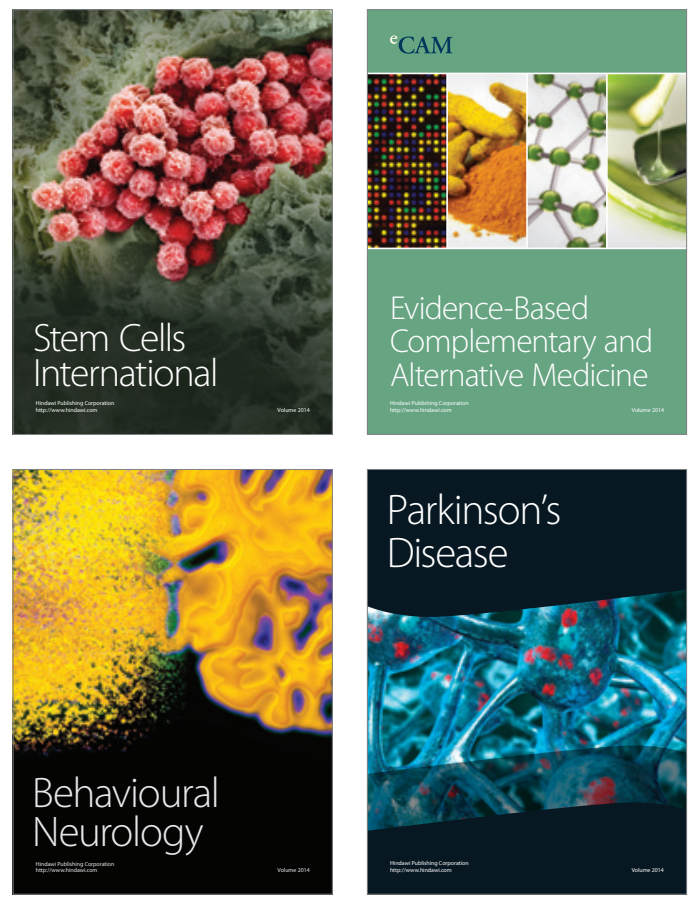
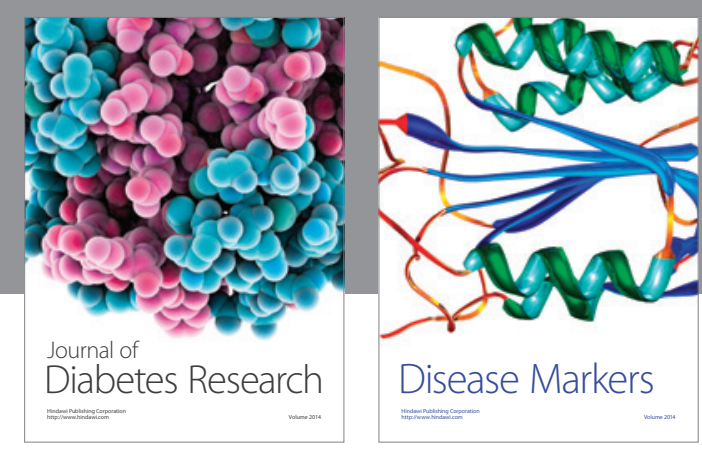

Disease Markers
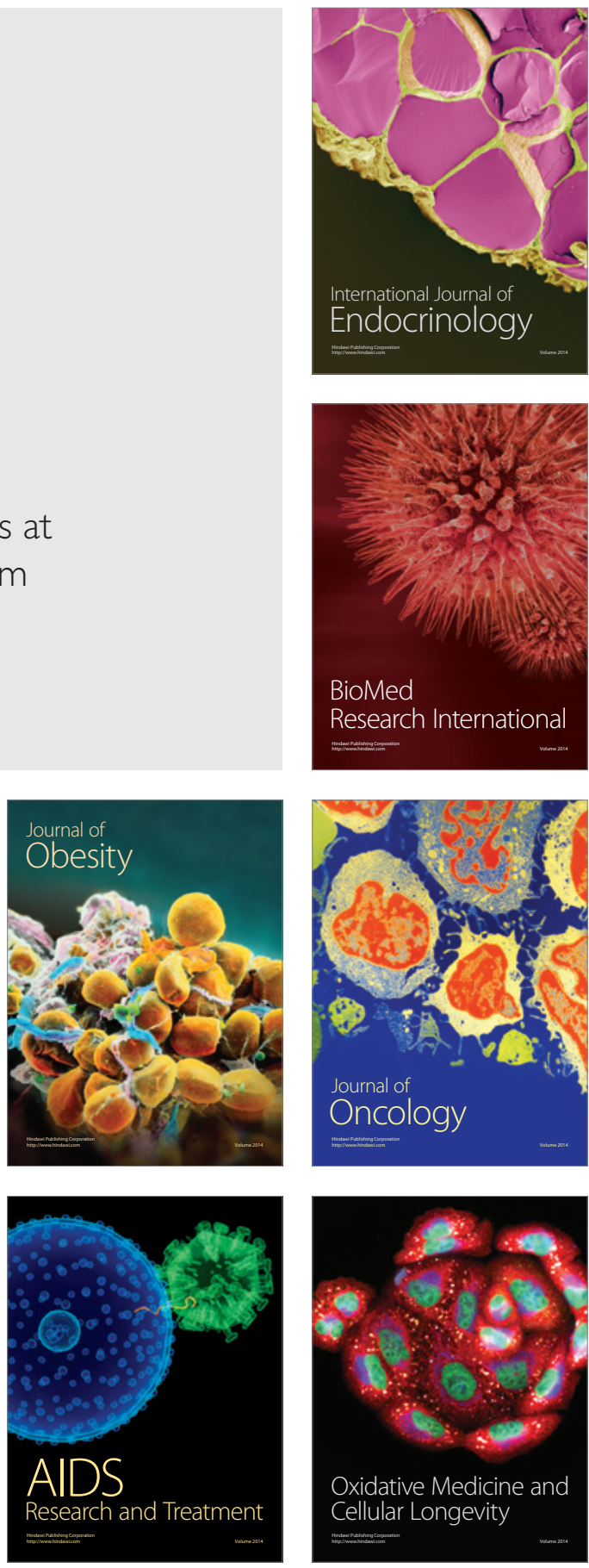